\title{
PIVKA-II as a Potential New Biomarker for Hepatocellular Carcinoma - A Pilot Study
}

\author{
SARKA SVOBODOVA ${ }^{1,2}$, MARIE KARLIKOVA ${ }^{1}$, ONDREJ TOPOLCAN ${ }^{1}$, LADISLAV PECEN $^{1}$, \\ MARTINA PESTOVA ${ }^{1}$, OTTO KOTT ${ }^{3}$, VLADISLAV TRESKA ${ }^{4}$, DAVID SLOUKA $^{1}$ and RADEK KUCERA ${ }^{1}$ \\ Departments of ${ }^{1}$ Immunochemistry, and ${ }^{4}$ Surgery, \\ University Hospital and Faculty of Medicine in Pilsen, Charles University, Czech Republic; \\ ${ }^{2}$ Third Internal Medicine Department and First Medical Faculty, Charles University, Prague, Czech Republic; \\ ${ }^{3}$ Faculty of Health Care Studies, University of West Bohemia, Pilsen, Czech Republic
}

\begin{abstract}
Aim: The aim of this study was to evaluate the clinical contribution of protein induced by vitamin $K$ absence (PIVKA-II) for hepatocellular carcinoma (HCC) diagnostics and compare it with alpha-foetoprotein (AFP), a routinely used tumour marker. Materials and Methods: A total of 332 participants were enrolled in this study: 64 with HCC, 48 with liver metastases of colorectal cancer origin, 42 with liver cirrhosis and 178 healthy individuals. Serum levels of PIVKAII were measured using the chemiluminescent assay of the Architect 1000i System (Abbott, USA) and AFP levels using the chemiluminescent assay by DxI 800 (Beckman Coulter, USA). Results: PIVKA-II achieved better clinical sensitivity than AFP and the difference in this sensitivity was statistically significant. PIVKA-II achieved the best sensitivity (96.9\%) in distinguishing between the HCC and control groups with the proposed cut-off value of $60 \mathrm{mAU} / \mathrm{ml}$. Conclusion: Our recommendation is for addition of PIVKA-II to the routine panel of HCC tumour markers.
\end{abstract}

Hepatocellular carcinoma (HCC) is the fifth most common cancer worldwide. Its survival rate does not exceed $15 \%$ (1). It is one of the leading causes of cancer-related deaths worldwide (2). The prevalence rates differ based on ethnicity. Higher rates are observed in Asian countries. It represents the second most common malignancy in China, but its incidence rate has been increasing in the more

This article is freely accessible online.

Correspondence to: Radek Kucera, Ph.D., Associate Professor, Department of Immunochemistry, University Hospital Pilsen, Edvarda Benese 13, 30599 Pilsen, Czech Republic. E-mail: kucerar@fnplzen.cz

Key Words: PIVKA-II, AFP, hepatocellular carcinoma, biomarkers, predictive. developed countries during the past few decades. Its mortality is almost equal to its morbidity (3). Carcinogenesis of HCC is multifactorial and includes previous infection with hepatitis B or C viruses (HBV, HCV), aflatoxin B or alcohol consumption resulting in liver cirrhosis (4). New cancer sometimes develops with no previous link to the abovementioned agents, sometimes non-alcoholic steatohepatitis in diabetic patients has been associated with liver cancer (5).

Serum alpha-foetoprotein (AFP) has been routinely used as a tumour biomarker for HCC detection and for monitoring the disease course. But it has not yet become an optimal biomarker for diagnostic purposes. Therefore, there is great interest in identifying and proposing new biomarkers to this end.

Protein induced by vitamin K absence (PIVKA-II), also known as des-gamma-carboxyprothrombin, has been described in relation to HCC (6). It is released in association with vitamin K deficiency and in the presence of HCC (7).

The aim of this pilot study was to evaluate the clinical contribution of PIVKA-II as a new biomarker for HCC diagnostics and compare it with the importance of AFP, a tumour marker routinely used in clinical practice.

\section{Materials and Methods}

Groups of patients. A total 332 participants were enrolled in this study. Exclusion criteria were the following: haemocoagulatory disorders, vitamin $\mathrm{K}$ uptake disorders, intake of vitamin $\mathrm{K}$ blocking agents, second cancer, acute inflammatory disease, renal and liver failure. The major cancer group with HCC included 64 patients. All clinical stages were included, but advanced stages were more frequent as shown in Table I. The second group comprised 48 patients with liver metastases of colorectal cancer origin. The third group of patients with benign liver diseases included 42 patients with liver cirrhosis resulting mostly from previous alcohol intake. The control group consisted of 178 healthy individuals who underwent a complete medical examination at the Department of Preventive Cardiology; at the time of blood collection, there was no evidence of any cancer or inflammatory disease. The list of the patient groups with age characteristics is shown in Table I. 
Serum samples. Serum samples were collected at the time of the diagnosis prior to any kind of therapy. Samples of peripheral venous blood were collected using the VACUETTE blood collection system (Greiner Bio-one Company, Kremsmünster, Austria). Serum was separated by 10 -min centrifugation at $1,300 \times g$ and immediately frozen to $-80^{\circ} \mathrm{C}$. Samples were thawed only once, immediately prior to analyses.

Methods used. Serum levels of PIVKA-II were measured using the chemiluminescent assay of the Architect 1000i System (Abbott, Libertyville, IL, USA) with limits of detection and quantification of 1.45 and $5.06 \mathrm{mAU} / \mathrm{ml}$, respectively; serum levels of AFP were measured using the chemiluminescent assay of the DxI 800 (Beckman Coulter, Brea, CA, USA) with limits of detection and quantification of 0.41 and $1.65 \mathrm{IU} / \mathrm{ml}$, respectively. Other laboratory tests were performed in order to rule out the presence of clinical conditions that may affect the serum level of the measured parameters (i.e. serious renal or liver failure, inflammation).

Statistical methods. Statistical analysis was performed using S.A.S. software (Statistical Analysis Software release 9.2; SAS Institute Inc., Carry, NC, USA). Basic descriptive statistic parameters are presented (median, minimum and maximum). Cut-off values were calculated at a $95 \%$ level of specificity. The Wilcoxon two-sample test was used for comparison of individual parameters and a $p$-value of less than 0.05 indicated statistical significance.

\section{Results}

Measured results of PIVKA-II and AFP are shown in Table II. Both biomarkers were found at the highest serum levels in the group of patients with HCC and the lowest levels were found in the control group. The optimal cut-off values at 95\% specificity are shown in Table III for both biomarkers. Individual cut-off values were established for each assessed group. In general, PIVKA-II achieved much better clinical sensitivity than AFP and the difference in this sensitivity was statistically significant in all comparisons. The PIVKA-II sensitivity varied over the range $89.1-96.9 \%$. PIVKA-II achieved the best sensitivity (96.9\%) when distinguishing between the HCC and control groups with the proposed cutoff value of $60 \mathrm{mAU} / \mathrm{ml}$. The AFP sensitivity varied over the range $34.3-50.0 \%$. AFP achieved the best sensitivity (50.0\%) in distinguishing between the HCC group and the group with metastatic colorectal cancer with the proposed cut-off value of $6 \mathrm{IU} / \mathrm{ml}$.

\section{Discussion}

PIVKA-II was first described as a new biomarker in 1996 and has been used since the beginning of the 21 st century. This pilot study represents the first study conducted in the Czech Republic related to PIVKA-II, which reflects the low incidence rate of this cancer type both in the Czech Republic and Europe as a whole. Hence studies related to this biomarker are very rare. Only few articles were published in
Table I. Groups of participants included in this study.

\begin{tabular}{lcc}
\hline Group & $\begin{array}{c}\text { Number of } \\
\text { individuals }\end{array}$ & $\begin{array}{c}\text { Median age } \\
\text { (min-max), } \\
\text { years }\end{array}$ \\
\hline Hepatocellular carcinoma & 64 & $68.5(31-83)$ \\
$\quad$ Stage I & $6(9.4 \%)$ & \\
Stage II & $13(20.3 \%)$ & \\
Stage III & $17(26.6 \%)$ & \\
$\quad$ Stage IV & $28(43.8 \%)$ & \\
Metastatic colorectal carcinoma & 48 & $62.5(34-78)$ \\
Benign liver disease - liver cirrhosis & 42 & $61.5(34-80)$ \\
Control group of healthy individual & 178 & $60.0(24-86)$ \\
Total & 332 & \\
\hline
\end{tabular}

Europe between the years 2015-2018 (8-12). Our own results correspond very well with French $(2,8)$ and Italian study results (11). The authors similarly describe that sensitivity and specificity of PIVKA-II achieved very high values and were significantly higher than those for AFP. A completely different situation is described in Asian populations. Some Asian authors present either higher sensitivity and specificity of AFP or very similar sensitivity and specificity. These authors recommend PIVKA-II only for the optimization of early diagnostic processes. The difference between European and Asian populations might reflect a different etiopathogenesis of liver cancer. In the Asian population, it seems that viral hepatitis $\mathrm{B}$ or $\mathrm{C}$ is the dominant risk factor $(13,14)$, but the European population mostly develops liver cancer from steatohepatitis of various origins (alcohol, metabolic syndrome, etc.) (15).

In our pilot study, we observed that serum PIVKA-II levels of the HCC group were significantly higher than the levels of the control group, the group with benign liver disease and the metastatic colorectal carcinoma group. Serum levels of patients with benign liver disease were significantly lower than those of patients with HCC (Table III). These results are consistent with the published studies $(16,17)$. Our data show even higher sensitivity and specificity compared to the previously published data (18). Serum levels of PIVKA-II in the group of patients with metastatic colorectal carcinoma was very close to those of the control group. AFP achieved a maximum level of $6 \mathrm{IU} / \mathrm{ml}$, and if used together with PIVKA-II, and potentially with some type of biomarker like cytokeratin, it would enable either confirmation or exclusion of the metastatic origin of liver tumour. We should also note that in our study the median AFP level in HCC was lower than that in benign liver diseases. Based on these facts, we assume that PIVKA-II might be used for primary diagnosis of $\mathrm{HCC}$, as well as for differential diagnosis of liver metastasis. Early diagnoses are very important nowadays when advanced surgery techniques have 
Table II. Summary of the results for individual groups.

\begin{tabular}{|c|c|c|c|c|c|c|c|}
\hline \multirow[b]{2}{*}{ Group } & \multirow[t]{2}{*}{$\mathrm{N}$} & \multicolumn{3}{|c|}{ PIVKA-II (mAU/ml) } & \multicolumn{3}{|c|}{ AFP (IU/ml) } \\
\hline & & Median & Min. & Max. & Median & Min. & Max. \\
\hline Hepatocellular carcinoma & 64 & 7,727 & 23.23 & 300,000 & 6.50 & 1.00 & 118,140 \\
\hline Metastatic colorectal carcinoma & 48 & 33.75 & 12.51 & 325.50 & 2.00 & 1.00 & 6.00 \\
\hline Benign liver disease & 42 & 121.17 & 20.07 & 563.00 & 8.50 & 1.00 & 25.00 \\
\hline Control group & 178 & 30.09 & 8.15 & 88.50 & 3.00 & 1.00 & 10.00 \\
\hline
\end{tabular}

PIVKA-II: Protein induced by vitamin K absence; AFP: alpha-foetoprotein.

Table III. Optimal cut-off for biomarkers at $95 \%$ specificity.

\begin{tabular}{|c|c|c|c|c|c|}
\hline \multirow[t]{2}{*}{ Distinction between } & \multicolumn{2}{|c|}{ PIVKA-II } & \multicolumn{2}{|c|}{ AFP } & \multirow[t]{2}{*}{$p$-Value* } \\
\hline & Cut-off (mAU/ml) & Sensitivity (\%) & Cut-off (IU/ml) & Sensitivity (\%) & \\
\hline HCC vs. control group & 60 & 96.9 & 14 & 37.5 & $<0.0001$ \\
\hline HCC vs. metastatic colorectal carcinoma & 130 & 95.3 & 6 & 50.0 & $<0.0237$ \\
\hline HCC vs. benign liver disease & 250 & 89.1 & 21 & 34.4 & $<0.0001$ \\
\hline HCC vs. all groups & 290 & 93.8 & 15 & 34.3 & $<0.0001$ \\
\hline
\end{tabular}

HCC: Hepatocellular carcinoma; PIVKA-II: protein induced by vitamin K absence; AFP: alpha-foetoprotein. *Wilcoxon test.

been introduced into routine practice (19-21). The results of our study of PIVKA-II suggest that using this new biomarker could provide information on the likelihood of cancer with a high probability. Subsequent costly imaging methods would further provide information on the tumour site and the extent of disease prior to surgery.

Based on our results, we can conclude that neither of the biomarkers fulfils the necessary criteria for use in screening, but the high sensitivity and specificity of PIVKA-II might be of use as an additional tool for primary diagnostics of HCC. We can see further potential in the differential diagnostics between $\mathrm{HCC}$ and other benign liver diseases, as well as in the differential diagnostics between primary HCC and liver metastasis, although the mechanism of PIVKA-II production in HCC and other cancer types remains unclear.

This pilot study will continue by focusing on the early stages (I and II) of HCC in order to evaluate its potential use in early HCC detection. Correlation between the serum levels of biomarkers and histological findings will be also evaluated. Our next research will also focus on correlations between PIVKA-II and findings by imaging methods (e.g. ultrasound, positron emission tomography - computed tomography).

In conclusion, the newly-tested PIVKA-II achieved better sensitivity in our pilot study than AFP, traditionally used a marker of HCC. Our recommendation is to add PIVKA-II to the routine panel of HCC tumour markers.

\section{Acknowledgements}

This study was supported by Ministry of Health, Czech RepublicConceptual Development of Research Organization (University Hospital in Pilsen-FNPl, 00669806).

\section{References}

1 Siegel RL, Miller KD and Jemal A: Cancer statistics, 2016. CA Cancer J Clin 66: 7-30, 2016.

2 Yang JD and Roberts LR: Hepatocellular carcinoma: A global view. Nat Rev Gastroenterol Hepatol 7: 449-458, 2010.

3 El-Seraqg: Epidemiology of viral hepatitis and hepatocellular carcinoma. Gastroenterology 142: 1264-1273, 2012.

4 El-Seraqg: Molecular pathology in early hepatocarcinogenesis. Oncology 78: 157-160, 2010

5 Siriwardana R.C., Niriella M.A, Dassanayake A, Ediriweera D., Gunetilleke B., Sivasundaram T, de Silva J.: Association of Serum Ferritin with Diabetes and Alcohol in Patients with NonViral Liver Disease-Related Hepatocellular Carcinoma. Liver Cancer 6: 307-312, 2017.

6 Yu R, Ding S, Tan W, Tan S, Tanj Z, Xiang S, Zhou Y, Mao Q and Deng G: Performance of protein induced by vitamin $\mathrm{k}$ absence or antagonist-II (PIVKA-II) for hepatocellular carcinoma screening in Chinese population. Hepat Mon 15: e28806, 2015.

7 Lefrere LL and Gozin D: Use of des-gamma-carboxyprothrombin in retrospective diagnosis of hidden intoxication of anticoagulants. J Clin Pathool 40: 589, 1987. 
8 Sultanik P, Ginguay A, Vandame J, Popovici T, Meritet JF, Cynober L, Pol S and Bories PN: Diagnostic accuracy of desgamma-carboxy prothrombin for hepatocellular carcinoma in a French cohort using the Lumipulse(®) G600 analyzer. J Viral Hepat 24: 80-85, 2017.

9 Jang ES, Jeong SH, Kim JW, Choi YS, Leissner P and Brechot C: Diagnostic performance of alpha-fetoprotein, protein induced by vitamin $\mathrm{K}$ absence, osteopontin, dickkopf-1 and its combinations for hepatocellular carcinoma. PLoS One 11: e0151069, 2016.

10 Poté N, Cauchy F, Albuquerque M, Voitot H, Belghiti J, Castera L, Puy H, Bedossa P and Paradis V: Performance of PIVKA-II for early hepatocellular carcinoma diagnosis and prediction of microvascular invasion. J Hepatol 62: 848-854, 2015.

11 Caviglia GP, Abate ML, Gaia S, Petrini E, Bosco C, Olivero A, Rosso C, Ciancio A, Pellicano R, Saracco GM, Rizzetto M and Smedile A: Risk of hepatocellular carcinoma in HBV cirrhotic patients assessed by the combination of miR-122, AFP and PIVKA-II. Panminerva Med 59: 283-289, 2017.

12 Viggiani V, Palombi S, Gennarini G, D'Ettorre G, De Vito C, Angeloni A, Frati L and Anastasi E: Protein induced by vitamin $\mathrm{K}$ absence or antagonist-II (PIVKA-II) specifically increased in Italian hepatocellular carcinoma patients. Scand J Gastroenterol 51: 1257-1262, 2016.

13 Wang X, Zhang W, Liu Y, Gong W, Sun P, Kong X, Yang M and Wang Z: Diagnostic value of prothrombin induced by the absence of vitamin $\mathrm{K}$ or antagonist-II (PIVKA-II) for early stage HBV-related hepatocellular carcinoma. Infect Agent Cancer 12: 47, 2017.

14 Nguyen-Dinh SH and Do A, Pham TND, Dao DY, Nguy TN and Chen MS Jr.: High burden of hepatocellular carcinoma and viral hepatitis in Southern and Central Vietnam: Experience of a large tertiary referral center, 2010 to 2016 . World J Hepatol 10: 116$123,2018$.

15 Asrih M and Jornayvaz FR: Metabolic syndrome and nonalcoholic fatty liver disease: Is insulin resistance the link? Mol Cell Endocrinol 1: 55-65, 2015.
16 Abd EGI, Mossallam GI, Radwan NH, Elzawahry HM and Elhifnawy NM: Comparing prothrombin induced by vitamin K absence-II (PIVKA-II) with the oncofetal proteins glypican-3, Alpha feto protein and carcinoembryonic antigen in diagnosing hepatocellular carcinoma among Egyptian patients. J Egypt Natl Canc Inst 26: 79-85, 2014.

17 Ertle JM, Heider D, Wichert M, Keller B, Kuper R, Hilgard P, Gerken G and Schlaak JF: A combination of alpha-fetoprotein and des-gamma-carboxy prothrombin is superior in detection of hepatocelullar carcinoma. Digestion 87: 121-31, 2013.

18 Zakhary NI, Khodeer SM, Shafik HE, Abdel Malak CA: Impact of PIVKA-II in diagnosis of hepatocellular carcinoma. J Adv Res 4: 539-546, 2013.

19 Treska V, Fichtl J, Bruha J, Liska V, Kormunda S and Finek J: Liver resections for colorectal metastases in patients aged over 75 years. Anticancer Res 37: 1529-1533, 2017.

20 Kucera R, Topolcan O, Fiala O, Kinkorova J, Treska V, Zedníková I, Slouka D, Simanek V, Safanda M and Babuska V: The Role of TPS and TPA in the diagnostics of distant metastases. Anticancer Res 36: 773-777, 2016.

21 Ludvík J, Duras P, Třeška V, Matoušková T, Brůha J, Fichtl J, Lysák D, Ferda J and Baxa J: Portal vein embolization with contralateral application of stem cells facilitates increase of future liver remnant volume in patients with liver metastases. Cardiovasc Intervent Radiol 40: 690-696, 2017.
Received August 16, 2018

Revised September 18, 2018 Accepted September 21, 2018 